\title{
Der Wurmfortsatz als einziger Inhalt eines eingeklemmten Bruches.
}

Von I)r. I. P. Wolf in Rüdersdorf.

In-Bruchsack von Leistenbriichen sind schon dic versohiedensten Fingeweideteile gefunden worden: dic Gallenblase, ein Teil des Magens, die inmercn Genitalicn etc. Daß der Wurmfortsatz allein oder im $\mathrm{Zu}$ sammenliang mit dem Coecuin in den Bruchsack gclangt, kann auch keincswegr als etwas Seltenes bezcichnct werden, I)ab aber einmal der Wurmfortastz allein in den Brucksack schliipft, festgeklcmmt wird und so die Erselpeinumgen eines aingeklemmten Bruches auftreten, dus habe ich in der Literatur nicht finden konnen, und ich möchte deshalb dic nachfolgende Bcobachtung als Kuriosum mitteilen.

De: ₹ \%wölfjährige Kuabe J. Sk. sucht das Krankenhaus auf mit folgenden Angabe:11: Schon immer bestand bei Husten und Pressen eine Vorwölbung in untersten Bauchabschnitt, dic jedoch nie in den Hodensack heruuterkam. Vor einigen Tagen trat ganz, plötzlich, ohne bekannte Crsache, cine sclımerzhafte, nicht «urückbringbare Vorwölbung inı Berciche des Teistenkanals mit Vergrößerung der rcchten Hoden. sacklü̈lfte auf. Seitdein bestanden Schmerzen bei allen Bewegungen, und c8 war niolıt möglich, ,den Bruch wieder zurückzubringen". Er. brechen und Stuhlverhaltung bestand nicht; da aber die Schmerzen \%unahinen, ebenso wio dic Spannung im Hodensack, kommt der Junge \&wecks Operation.

Bcfund: Die rechte Skrotalhälfte ist prall gespannt. Einzelheiten an ihr sind nicht zu fuhlcn. Der Versuch, den Leistenkanal zu palpieren, vrweist sich als unmöglich und ist äußerst schmer\%haft. Iie Gegend des Leistenkanals erscheint ctwas vorgewölbt und ist druckempfindlich. In der Gegend des inneren Leistenringes glaubt man cinen sich hier nach innen fortsotzenden Strang zu fühlen. Der Befund war derselbe wic bei gleich \%eitigem Bestehen einer Hydrocele testis und Hydrocele funiculi spermatici; doch widersprach dieser Annahme die Entstehungs. geschichte und der Schmor\%.

Operation in Narkose: Nach Spaltung der Haut und der weiteren Schichten pröffnct man einen mit trüber, flockiger F'liisaigkeit prall gefüllten, glattwandigen Hohlraun, in dessen Grunde skrotalwärts der Hoden liegt. Leistenkanalwärts besteht ein völliger Abschluß 'durch einell sehr bald als den peripherisehen Teil der Appendix erkannten, $3-4 \mathrm{~cm}$ langen, mit fest an sie angedrücktem Mcsenteriolum in den Hohiraun hincinragenden Zapfeu. Die Appendix licB sich weder vorzichen, noch auch \%urückschieben. Dics gelang erst nach Spaltung der cinklemmenden äußcren Leistenkanalöffinung. Verwachsungen bestanden nicht. Der äußerc Lcistenring und Bruchsackhals hatte die Weite cines dünnen Bleistifts; der Loistenkanal war im ïbrigen wesent. lich witer, Der Bruchsack sclbst war sehr zart. Es wurde nun die Appendix cktoniert und dic: Radikaloperation des Leistenbruchs nach Riedel vorgenommen. Dic Heilung war ungestört. - Das Präparat der etwa $12 \mathrm{~cm}$ langen Appendix zeigte an der Stelle der Umachnirrung, vor allem an der Sehleimhaut Zeichen der Einklemmung. Die Serosa des peripherisehen geschwollenen Appendixteiles war fibrinbedeckt.

Wir haben es also hicr mit einem Fall von Finklemmung der Appendix in einem angeborenen rechtseitigen Leistenbruch zu tun. Dic voll dem Patienten in frïherer Zcit gelegentlich bcobachtete ,Vorwölbung in unterstken Banchsbachnitt, dic durch le.ichten 1)ruck mit der Hand beseitigt werden kounte," war zweifellos bedingt dureh das Eindringen von Bauchinhalt in den I cistenkanal. Dic Möglichkeit, dic äußere, relativ engo Lcistenkanalöffnung mit dem engen Bruchsackhalsc zu passieren, bestand nur bei langem Mesocoecum für den Wurmfortsatz und auch für dicsen nur bei starkeın Pressen o. dgl. mit den Folgen de: Einklemmung. 